\title{
Collaborative Crafting of Interlocking Structures in Stereotomic Practice
}

\author{
Shayani Fernando ${ }^{I}$ \\ ${ }^{I}$ The University of Sydney \\ 1shayani.fernando@sydney.edu.au
}

\begin{abstract}
Situated within the art of cutting solids (stereotomy) and the evolution of machine tools; this research will investigate subtractive fabrication in relation to robotic carving of stone structures. The advancement of the industrial revolutions in the mid to late 19th century saw the rise of new building techniques and materials which were primarily based on structural steel construction. The modern aesthetic of the time further diminished the place of traditional stonework and ornamentation in modern structures within the building arts. This paper will focus on the design and fabrication of three sculptural dry-stone modular prototypes investigating interlocking self-supporting structures in stone. Examining the value of robotic technologies in the design and construction process in relation to collaborative crafting of the hand and machine.

Accommodating for material tolerances which are a major factor in this research. Interrogating the value of robotic crafting with material implications and exploring the role of the artisan in machine crafted architectural components.
\end{abstract}

Keywords: Collaborative, Crafting, Interlocking, Structures, Robotic Fabrication, Digital Stone

\section{INTRODUCTION}

Subtractive methods that cut and carve away material have been developed throughout history from Neolithic times. (Garrison, 1999) With the recent advances in Computer Aided Design and Manufacturing CAD/CAM technologies, the processes can be achieved with more precision and efficiency, (BrellÇokcan, Braumann, 2013). Manual reduction of large-scale stone structures is often difficult and labour intensive with traditional knives and blades. Developments in mechanistic operations due to industrialisation have amplified and refined this process of subtraction. (McGee et al. 2013). Human engagement within this manufacturing process in- cludes decision making in the initial design phases, material choices and final manufactured outcome.

This paper extends previous research into the development of fabrication options using ruled surface geometry for interlocking wave joints, "Stereotomy of Wave Jointed Blocks" (Weir et al. 2016) and "The Culture of Crafting: Exploring the relationship between the Hand and the Machine in Digital Stone Sculpture" (Fernando, 2018). The focus of this research, however, will be to question the value of collaborative crafting and the necessity of the human within robotic fabrication workflows, for designing and constructing self-supporting structures within the field of stereotomic practice in the age of 
the 4th industrial revolution. As described by McCullough industrialisation made "practices like craft something no longer to be taken for granted, and further degraded the stature of the artisan." (McCullough, 1996) However, industrialisation has influenced stone crafting techniques where the 'artisan' or stone mason must now acquire new skills to work with developing automated machine technologies. Suggesting the cultural shift in the role of the artisan and methods of crafting.

\section{STEREOTOMIC PRACTICE}

There exists a gap in the practice of stereotomy as a result of the first and second industrial revolutions. According to the world economic forum, "The First Industrial Revolution used water and steam power to mechanize production. The Second used electric power to create mass production. The Third used electronics and information technology to automate production. Now a Fourth Industrial Revolution is building on the Third, the digital revolution that has been occurring since the middle of the last century."(Schwab, 2016) Although this description provides a simplified explanation into the formation of the fourth industrial revolution, it does not articulate what occurs in subtractive manufacturing fields and the value of crafting.

Schwab later in the article suggests, "Engineers, designers, and architects are combining computational design, additive manufacturing, materials engineering, and synthetic biology to pioneer a symbiosis between microorganisms, our bodies, the products we consume, and even the buildings we inhabit." Whilst additive manufacturing processes of creating concrete and sandstone have become increasingly developed over the last few years, the research into their chemical composition and sustainability can be further studied. An alternative solution is subtractive manufacturing using material that already exists. Therefore, the field of stereotomy (the art of cutting solids) has re-emerged with evidence of dry stone structures from Neolithic times which have survived centuries.
The return of stereotomy is largely due to the improvement of representational tools, with the application of these representational tools the material problems of the stone mason has become the pathway to revive the stalled tradition of stone architecture. "Once a slow and painstaking process of cutting each stone by hand, now stone masonry can proceed rapidly with computer-guided cutters that can fashion more complex shapes than a person working manually with hand-held tools. And computer graphics enable architects to explore sophisticated forms while subjecting them to static analysis for safety' (Etlin, 2008).

\section{Hand and Machine Crafting methods}

Historically master stone craftsman and sculptors had acquired the instructional skill through the constant refinement of their craft which was then communicated through models or inscription onto the stone material itself usually at 1:1 scale. The 'master builder' who would be involved in making decisions about the application of geometry and selection of material. The material itself give much meaning to the final result as the formal design method used. For example in the selection of stone to carve the statue of David from 1501-1504 during the Renaissance period, Michelangelo obtained a large block of Carrara marble quarried forty years earlier in the Apuan Alps of northern Tuscany. The marble had to be of "good quality, white and beautiful, and without faults,"(Wallace, 1994). To an architect or sculptor's eye, some "visually attractive features of stone blocks are flaws, leaving them structurally fragile"(Fernando et al, 2015). Thus the carving away of a block of stone also requires knowledge into the material properties and many master stone craftsman and masons have this knowledge through years of acquired skill.

Whereas previously manual cutting stone to precise measurement epitomized a laborious task (Burry, 2016) the integration of automated workflows early in the design process enables efficient machining practices contributing to changes in the construction industries and adding value to digital crafting 
Figure 1

Left: 7 axis robotic carving with the saw blade, Middle: Robotic milling of marble at Garfagnana Innovazione, Right: Abrasive wire saw cutting sandstone at Gosford Quarries
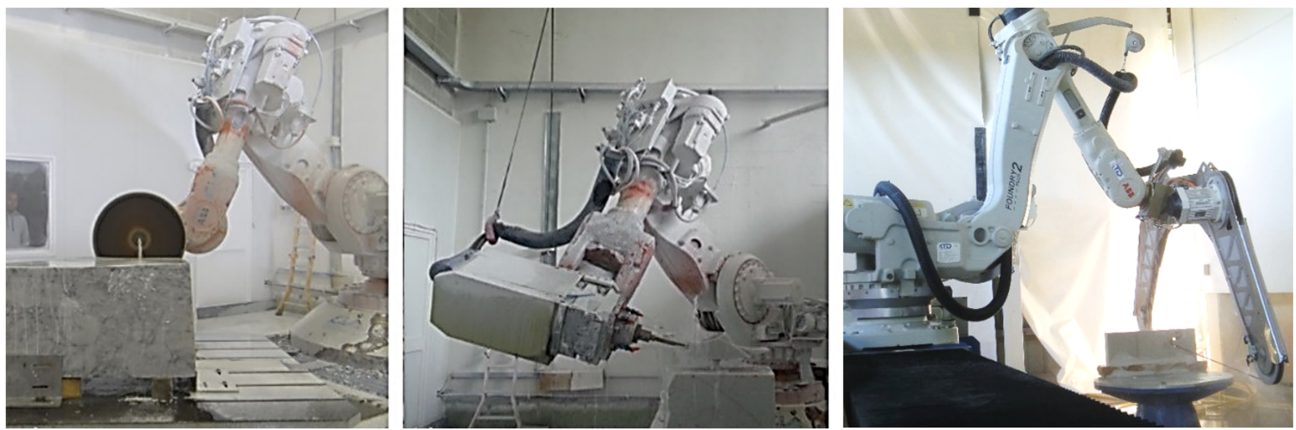

methods. This is due to the recent advancements in software technologies from 3D modelling and scripting environments to machine code. Material integration with structural analysis and advanced subtractive fabrication techniques of cutting stone modules are being explored by several research groups such as the Block Research group, New Fundamentals Research group, Carrara Robotics, Odico and Matter Design Studio. The focus of this research, however, is on the design and fabrication of three sculptural prototypes in relation to fabrication workflows and crafting.

\section{INTERLOCKING STRUCTURES}

Interlocking assemblies in the construction industry have gained acceptance for their versatility and reduced labour costs in comparison to traditional brick and mortar methods. The ease of assembly and disassembly make using these mortar-less interlocking blocks for arched and vaulted spaces appropriate. As described by several researchers (Fallacara, 2019)(Vella \& Kotnik, 2016)(Estrin, 2014), the relationship of stereotomy to topological interlocking blocks was developed in the flat vault structure (Abeille's vault) invented by Joseph Abeille in 1699. Estrin explains that the "medieval design known as the Abeille's vault, which is based on what we would now call topological interlocking of trun- cated tetrahedra....('Abeille-like') and osteomorphic blocks ('Truchet-like'), are highly tolerant to local failures." (Estrin, 2014) The use of interlocking wave joints (Weir et al, 2016) are used for the following explorations in stereotomic crafting of self-supporting stone structures.

\section{STEREOTOMIC PROTOTYPES}

In the context of stereotomic practice, advanced fabrication of interlocking wave geometry with wirecutting, waterjet and CNC milling processes has opened new possibilities for crafting stone modules with precision and efficiency. In relation to the specific prototypes produced during the digital stone project workshop, the artists have "repurposed software and machinery designed for the aerospace and automotive industries to the humanistic purpose of art." (Isherwood, 2017)

The machines utilised to cut and craft the material to the model specifications included a 7 axis $A B B$ Foundry robot arm with various end effectors. These end effectors included a saw blade (Figure 1 and 2), and diamond coated CNC milling tools (Figure 1), to different sizes and thickness. The 7th axis was the turntable in which the block of marble was mounted with timber and plaster bases. The saw blade was attached at the roughing stage of the block followed by the carving CNC milling tools. Whilst the geome- 
try was modelled as ruled surfaces which can be fabricated by an abrasive wire saw (Figure 1), the available machine was the CNC 7 axis milling robot which produces more waste than the abrasive wire saw. However, it was observed that the remaining marble dust from the machine goes into another facility which manufactures synthetic or composite stone types. The following will describe the process of the design and fabrication of three sculptural prototypes based on wave jointed blocks developed to 'Catenary Tales' and 'Archi-Twist'.

\section{Design and Fabrication of Wave Jointed Blocks}

The waveform prototype joint design was developed through a process of iterative drawing which was then parametrised in RHINOCEROS and GRASSHOPPER. One of the most significant aspects of this geometry is that it is made from 'ruled surfaces' to accommodate fabrication methods with robotic wire cutting. As a comparison study into EPS foam blocks and natural stone blocks (Fernando et al, 2017), two similar geometries were fabricated with Gosford quarries (Figure 2). This prototype demonstrates both potential for column and cantilever structural conditions.

These two blocks of interlocking wave geometry were simplified from the initial three blocks proposed in the 3D model have a very similar workflow to the following prototypes 'Catenary-Tales' and 'Archi-Twist' in that a saw blade and CNC milling were used as the main machines to process the sandstone block. The model as shown (Figure 2) initially comprised of 3 blocks to be cut and carved, however, due to machine time limitations, this was cut to 2 blocks. Each block at scale 1:2 weighed approximately 12-15 $\mathrm{kg}$ and took 4 hours with 3 tool changes each. Minimal hand sanding was applied at the final stage as a finer abrasive diamond tool was used specifically for the finishing stage.

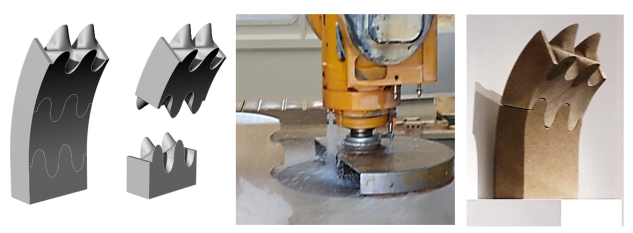

\section{Design and Fabrication of 'Catenary Tales'}

The concept for the development of 'Catenary Tales' (2015) stemmed from an exploration of selfsupporting structures in natural stone. The sculptural prototype is made from interlocking wave joinery in varying sizes of modules which engages with the tensile strength of stone. The production process involved the design in Sydney using 3D modelling software RHINOCEROS and manufactured as part of the Digital Stone Project workshop at Garfagnana Innovazione in Italy using 7-axis robotic carving machines.

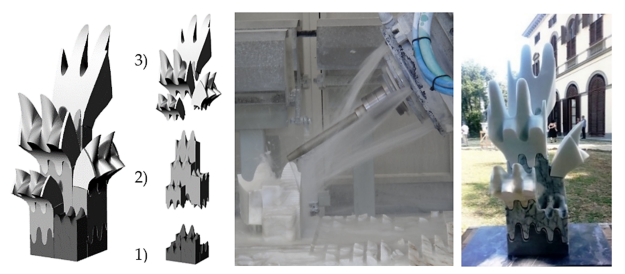

It aims to reform the value of 'crafting' through innovative manufacturing technologies and sustainable natural stone. A combination of machined and hand finishes were applied (Figure 3 ) to the sculptural prototype. The overall dimensions are $38 \times 36 \times 80 \mathrm{~cm}$ and weighing at $60 \mathrm{Kg}$ in total. The marble type is similar to the colours chosen for 'Archi-Twist' which include Bianco Acquamarina, Venato Orto di Donna and Bardiglio Imperiale. The darker colours at the base while the lighter Carrara marble at the top which further contributed to highlight the complexity of the geometry and material.
Figure 2

Left: 3D model

render, Middle:

Fabrication of 2

modules with the

saw-blade, Right:

Exhibited

sandstone

prototype at the Tin

Sheds Gallery,

Sydney

Figure 3

Left: 3D model render, Middle:

Fabrication order, machine and assembly method,

Right: Final exhibited Sculptural prototype at the Marble Codes

Exhibition in

Florence 2015 
Figure 4

Left: 3D rendering of model, Middle: cut blocks of stone ready for hand finishing and assembly, Right: Exhibited Sculpture at the Digital Stone Project exhibition 'Metamorphic Resonance' in Pietrasanta 2017.
The exhibited prototype 'Catenary Tales' was successful in that through the collaboration of the hand and the machine, the realisation of the initial 3D model was achieved. The specific factors which made it successful include the skill of the craftsman in order to remediate the machine inconsistencies and performance with the material. The skill of the fabrication team and technicians to realise the 3D model into physical reality involved many attempts especially if there were cracks and fractures in the material. The workflow of both the modelling and fabrication process could have further integrated methods of material surveying and scanning. However, the focus was on developing the skill of crafting and carving away material from natural stone.

Constraints included a limited 25-hour machine time for each prototype which affected both the final scale and number of machined parts. The initial modelling process, however, could have benefited from a more systematised approach to both the structural analysis and modelling which was based on topological modelling of scaled interlocking blocks. It can be argued that the sculptural qualities and irregularities which make the final product so interesting were attributed to the inconsistencies in the model and machining process, together with material flaws. The very controlled modelling process of the 'Archi-Twist' prototype also included these machine and material inconsistencies.

\section{Design and Fabrication of 'Archi-Twist'}

The concept of the 'Archi-Twist' prototype designed and manufactured as part of the Digital Stone Project is a twisted catenary arch comprised of innovative modular interlocking wave joinery based on catenary curvature. The method of geometry generation was developed in an entirely parametric environment. In contrast to using sinusoidal or interpolated curvature (used in the initial wave jointed block geometry), catenary curvature for the interlocking wave amplitude has a higher contact surface area thus facilitating a better interlocking capacity. Furthermore, the 180-degree twist of the bases of the arched struc- ture was modelled for both aesthetic and structural reasons due to the extent of the twisting capacity of ruled surface joints. It not only tested the potential capacity of the wave joint contact surfaces but also provided a stabilising mechanism for the overall macro geometry of the arch (Figure 4).
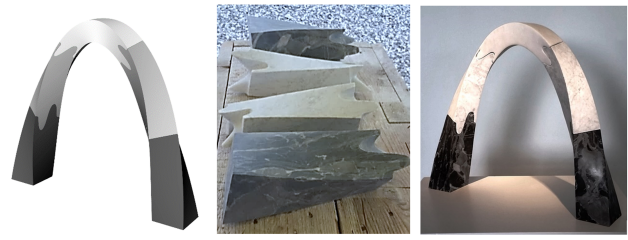

Due to limitations in machine time which were 25 hours per project within the workshop setting, the scale and number of blocks to be fabricated had to be reduced from 7 parts to 5 . Each part (Figure 4) was machined in approximately 5 hours including tool changes. However, this was still not enough as a final finished module as there was usually 1$2 \mathrm{~cm}$ extra material left to sand by hand using the power tools, grinders and hand sanding paper processes (Figure 5) similar to processes used by traditional marble sculptors. The overall dimensions of 'Archi-Twist' were $14 \times 66 \times 56 \mathrm{~cm}$, weighing at $38 \mathrm{~kg}$ in total.

\section{Toolpath generation in crafting process}

With the introduction of six-axis robotic fabrication methods, customised parts in complex shapes, semiautomated fabrication of modules, and the serialisation of structurally efficient joints become affordable and feasible. Factors such as robotic toolpaths can further be explored in relation to crafting techniques. Whilst the toolpaths for wire cutting were based on a two-pass sequence to cut the block of stone or foam based on efficiency and minimal wastage of material, CNC milling toolpaths varied. In fact, the toolpaths generated for many of the prototypes during the Digital Stone Project workshop were integrated as part of the design aesthetic to express the char- 

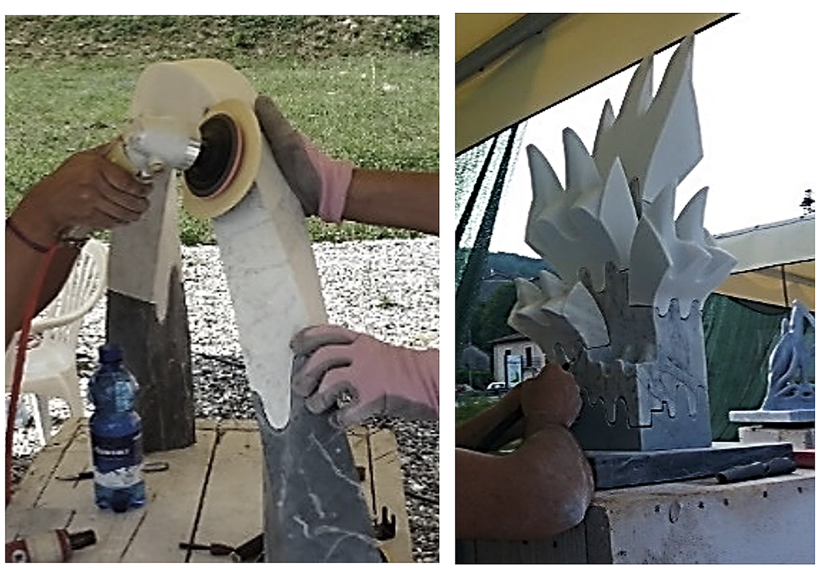

Figure 5

Hand Sanding,

finishing and detailing processes with professional sculptors after the machine cuts acteristics of the toolpaths. In the specific cases of the prototypes 'Archi-Twist' and 'Catenary Tales' the robotic toolpaths were generated to allow for maximum machine and material tolerances to minimise fractures in the more delicate and thinner areas of the model. These toolpaths become part of the 'crafting' method of the machine and the modeller. The use of the machine tools is of assistance to the workflow and process of crafting rather than being a replacement for handcrafting techniques.

\section{MATERIAL TOLERANCES}

Tolerances are a major consideration to implement in the early design phases. As the modules are aggregated and assembled, their material and structural properties become relevant in the manner of assembly and stability. It was found that the assembly of just 2 blocks into a column or part cantilevering structure was quite complex and dependant on the material strength, density and geometry. EPS foam has a higher tolerance than natural stone for modules to fit together. One observation was that sandstone processing took slightly less time than marble at the same scale of CNC milling 1:2 scale blocks. This is due to the lower values in density and compressive strength. The machine and method of cutting further implicate tolerances in gaps between contact surfaces. Generally, a 1-2cm tolerance gap was modelled in before fabrication to allow for the final finishing and fitting stages.

The advantage of this construction system is that the modules are small enough to be lifted by a human. Many of the stone sculptures produced in the Digital Stone workshop required the assistance of a forklift to manoeuvre the stone, whereas the smaller modular sculptures can be transported relatively easily by hand. Hand finishing and detailing were an important step in the crafting process where there were professional stone craftsman and sculptors to assist (Figure 5). One of the factors to consider in the transportation of these modules was how fragile the edges were. The sharp or thinner sections within the contact surfaces and outer edges of the structure can potentially chip away. This occurred also during the machining stage where small fractures in the stone were repaired using glue. In order for the parts to fit together, the use of the carbon copy method (red chalk on stone) by sculptors was employed to find gaps within the contact surfaces. Power tools such as drimmels, sanders and small grinders were utilised to finish the prototype which was then polished for the exhibition. 
The significance of the hand finishing process is heightened as the need for the blocks to fit together is of primary importance for these prototypes. The freshly cut machined parts did not automatically fit together as there was extra material to accommodate for machine and material errors. The collaboration of the hand and the machine is what makes this project retain its artisanal qualities with the skill of hand finishing marble (Figure 5). Professional sculptors were on hand to both teach and assist in the final finishing stages so that the less experienced artists and designers can learn their craft of hand finishing and final detailing. It also enabled the artists more time to 'feel' the stone and understand how it behaves and reacts to certain conditions.

\section{CONCLUSION}

To reflect on this work, the initial aim of this research was to generate new knowledge manifested in the crating of a specific geometry of interlocking modular arch structures in natural stone utilizing robotic technologies. The specific geometry in the form of a wave block module was then aggregated to form an architectural structure and/or space. The success of the outcome was determined by the relevance and usefulness of the generated knowledge through structural constraints, material efficiency and strength. A series of material prototypes were created and exhibited both locally and internationally. The paper discusses the importance of human labor and the artisan's role in the field of stereotomy during the fourth industrial revolution. The argumentation is based on a collaborative experience between the human hand and machine at the production of three prototypes sculpted with a seven-axis robotic arm. A finalized prototyping report of the three prototypes is outlined and its reflection on the contribution of human labor in an automated sculpting process.

This research has established the design criteria and constraints of the designed interlocking modules including artisanal qualities and design intentions related to stone crafting. "Artisans of pre-industrial times were not engineers; hence they did not use mathematics to predict the behaviour of the structures they made. When they had talent they learned intuitively, by trial and error" (Carpo, 2017). This statement raises the question of how to integrate artisanal knowledge into emerging robotic fabrication techniques formed by the 4th industrial revolution. This research has shown that collaborative workflow between the hand and the machine is required for effective, crafted and valued architectural outcomes.

\section{Future Work}

The use of interlocking structures in the construction industry has the potential to shape the design and fabrication process due to efficient assembly and disassembly techniques. This is, however, variant on the geometry and framework of the method. An algorithmic approach to the generation of interlocking structures as proposed by Wang et al, 2018 is based on representing "part relationships with a family of base Directional Blocking Graphs and leverage efficient graph analysis tools to compute an interlocking arrangement of parts." This facilitates a more complex arrangement of parts which can be applied to various materials such as timber. However the issue of tolerances discussed in the prototypes above, still apply and have to be accounted for. The extension of this research to be applicable to other materials such as timber further highlights the relevance of stereotomic practice in architecture within the 4 th industrial revolution.

\section{Acknowledgements}

The author would like to express her gratitude to the Digital Stone Project organisers, Garfagnana Innovazione, Gosford Quarries and University of Sydney advisors, DMaF lab staff for the opportunity to participate in the workshops with the assistance of University of Sydney research grants, ACIS Cassamarca Research Scholarship and Autodesk scholarship for the DSP workshop. 


\section{REFERENCES}

Brell-Çokcan, S and Braumann, J 2013, 'Introduction.', in Brell-Çokcan, S and Braumann, J (eds) 2013, Rob|Arch 2012: Robotic Fabrication in Architecture, Art, and Design, Springer, Vienna, pp. 8-11

Burry, M 2016, 'Robots at the Sagrada Familia Basilica: A Brief History of Robotised Stone Cutting', in Reinhardt, D, Saunders, R and Burry, J (eds) 2016, Robotic Fabrication in Architecture, Art and Design (2016), Springer International Publishing, Switzerland, Vienna, pp. 3-17

Carpo, M 2017, The Second Digital Turn: Design Beyond Intelligence, MIT Press, Cambridge MA

Dyskin, A. V., Estrin, Y and Pasternak, E 2019, 'Topological Interlocking Materials', in Estrin, Y, Bréchet, Y, Dunlop, J and Fratzl, P (eds) 2019, Architectured Materials in Nature and Engineering, Springer Series in Materials Science, Switzerland, pp. 23-49

Estrin, Y 2014, 'Topological Interlocking and Osteomorphic Blocks', in Fallacara, $\mathrm{G}$ and Minenna, V (eds) 2014, Stereotomic Design-Catalogo della mostra omonima per Inside Marmomacc and Abitare il Tempo Veronafiere, Maglie, Verona, pp. 11-13

Etlin, R 2008, 'Stereotomy, Acrobatic Stone Vaulting Past and Future', in Etlin, R, Fallacara, G and Tamboréro, L (eds) 2008, Plaited Stereotomy; Stone Vaults for the modern world, ARACNEeditrice S.r.l., Rome, p. 17

Fallacara, G, Barberio, M and Colella, M 2019, 'Topological Interlocking Blocks for Architecture: From Flat to Curved Morphologies', in Estrin, Y, Bréchet, Y, Dunlop, J and Fratzl, P (eds) 2019, Architectured Materials in Nature and Engineering, Springer Series in Materials Science, Switzerland, pp. 423-445

Fernando, S 2018 'The Culture of Crafting: Exploring the relationship between the Hand and the Machine in Digital Stone Sculpture', Proceedings of the 1st Annual Design Research Conference, Sydney 2018, University of Sydney, pp. 509-516

Fernando, S, Reinhardt, D and Weir, S 2017 'Waterjet and Wire-Cutting Workflows in Stereotomic Practice: Material Cutting of Wave Jointed Blocks', Protocols, Flows and Glitches. 22nd International Conference for Computer-Aided Architectural Design Research in Asia (CAADRIA 2017), Suzhou, China, pp. 787-798

Garrison, E. G 1999, A history of engineering and technology: artful methods, CRC Press, New York

Isherwood, J and Coiai, S (eds) 2017, Metamorphic Resonance (Digital Stone Project 2017 Exhibition catalogue), Garfagnana Innovazioni, Pietrasanta
McCullough, M 1996, Abstracting craft: the practiced digital hand, MIT Press, Cambridge, MA

McGee, W, Feringa, J and Søndergaard, A 2013, 'Processes for an Architecture of Volume', in BrellÇokcan, S and Braumann, J (eds) 2013, Rob | Arch 2012, Springer, Vienna, pp. 62-71

Vella, I. M. and Kotnik, T 2016 'Geometric Versatility of Abeille Vault', Proceedings of eCAADe 2016, Finland, pp. 391-397

Wallace, W.E. 1994, Michelangelo at San Lorenzo: the genius as entrepreneur, Cambridge University Press, London

Wang, Z, Song, E. P. and Pauly, M 2018, 'A General Framework for Designing Interlocking Assemblies', ACM Transactions on Graphics, 37(6), p. 191

Weir, S, Moult, D and Fernando, S 2016, 'Stereotomy of Wave Jointed Blocks: Toward a Wave-Jointed Stone Construction Using Wire Cutter Toolpath Generation., in Reinhardt, D, Saunders, R and Burry, J (eds) 2016, Robotic Fabrication in Architecture, Art and Design 2016, Springer, Switzerland, pp. 284-293

[1] https://www.weforum.org/agenda/2016/01/the-fou rth-industrial-revolution-what-it-means-and-how-to-re spond/ 